\title{
PARAMETRIZATION AND RANGE OF MOTION OF THE BALL-AND-SOCKET JOINT
}

\author{
Paolo Baerlocher and Ronan Boulic \\ Computer Graphics Lab (LIG), Swiss Federal Institute of Technology (EPFL), 1015 Lausanne \\ Switzerland
}

Key words: Ball-and-socket joint, joint limits

\begin{abstract}
The ball-and-socket joint model is used to represent articulations with three rotational degrees of freedom (DOF), such as the human shoulder and the hip. The goal of this paper is to discuss two related problems: the parametrization and the definition of realistic joint boundaries for ball-and-socket joints. Doing this accurately is difficult, yet important for motion generators (such as inverse kinematics and dynamics engines) and for motion manipulators (such as motion retargeting), since the resulting motions should satisfy the anatomic constraints. The difficulty mainly comes from the complex nature of 3D orientations and of human articulations. The underlying question of parametrization must be addressed before realistic and meaningful boundaries can be defined over the set of 3D orientations. In this paper, we review and compare several known methods, and advocate the use of the swing-and-twist parametrization, that partitions an arbitrary orientation into two meaningful components. Finally, we review two joint boundaries representations based on this decomposition, and show an example.
\end{abstract}

\section{INTRODUCTION}

In fields such as robotics [8] and biomechanics, and in Computer Animation as well [11], hierarchical structures are used to model articulated bodies like (real or imaginary) robots, humans and other creatures. An articulated body is made of a set of segments, connected by joints. The essential feature of a joint is that it permits some degree of relative motion 
between the two segments it connects. Ideal kinematic joint models are defined in order to formalize this permitted relative motion, called range of motion, characterized by the number of parameters that describe the motion space, and constrained by joint limits. Modeling real joints can be very complex, since the range of motion depends on many factors, especially in the articulations of living organisms and the human in particular [2]. Moreover, joints may be dependent on each other. In this paper, the coupling between joints is ignored.

The simplest example of joint model is the revolute joint that allows a rotation about an axis fixed in both segments it connects, usually within some angular limits. This joint is said to have one degree of freedom (DOF) and, because of its simplicity, is by far the most used joint in robotics. In human modeling, it is a convenient model of the interphalangeal joints of the hands and feet, for example. For more complex articulations such as the shoulder and hip, joint models allowing more degrees of freedom are required. The kinematic modelling of such articulations is a difficult task. First, a clear mathematical description of the allowed relative motion must be given by a proper parametrization: because of the complex non-Euclidean nature of rotations, this must be done carefully, because of the problem of singularities. Second, the range of motion must be constrained to restrict the parameter space to a more realistic subset. The problem is complex for balland-socket joints, because the boundaries on the three independent parameters are generally coupled.

In Computer Animation, these topics have already been addressed by Badler [1, 2], Korein [5], Wang [9, 10], Grassia [4] and Maurel [7]. In this paper, we summarize and compare their results, and try to provide some more insight on the topics.

\subsection{Notation and conventions}

Vectors are denoted by small boldface letters such as $\boldsymbol{v}$. The three basis vectors of a coordinate frame are noted $\boldsymbol{x}, \boldsymbol{y}$ and $z$. Matrices are denoted by capital letters such as $M$.

The rotation by an angle $\theta$ about an axis passing through the origin and whose direction is given by vector $a$, is noted $R_{a}(\theta)$.

Given two unit vectors $a$ and $b$, we define $R_{D}(a, b):=R_{a \rtimes b}\left(a \cos \left(a^{T} b\right)\right)$ as the direct rotation that transforms $\boldsymbol{a}$ into $\boldsymbol{b}$, with the minimum angle of rotation. If $b+a=0, R_{D}(a, b)$ is undefined. 


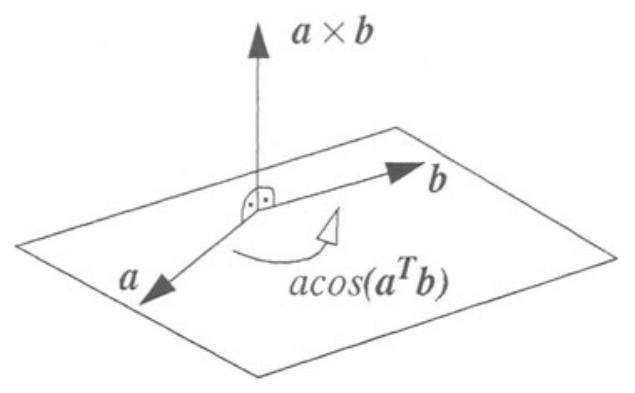

Figure 1. A direct rotation transforms a unit vector $\boldsymbol{a}$ into a unit vector $\boldsymbol{b}$.

\section{PARAMETRIZATION OF A BALL JOINT}

A ball-and-socket joint possesses three rotational degrees of freedom. Hence, it is the most mobile of the purely rotational joints. It allows an axial motion (or twist) of the segment (one DOF), as well as a spherical motion (or swing) that determines its direction (two DOFs). Ball-and-socket joints are used to model articulations such as the human shoulder and hip. By convention, the moving segment is aligned with the $z$ axis of the local joint frame (see Fig. 2).

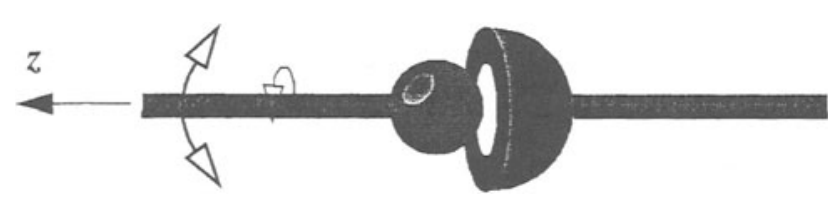

Figure 2. Mechanical illustration of a ball-and-socket joint.

\subsection{Parametrization of rotations}

The motion space of a ball-and-socket joint is the set of 3D rotations. There are many well-known parametrizations of rotations, such as: the Euler angles (the angles of three successive rotations about main axes), the unit quaternion (also known as the Euler parameters), the axis-angle vector (also known as the exponential map).

Good comparisons of such parametrizations for the purposes of animation of articulated bodies can be found in [4] and [11]. As noted by [4], no single parametrization of rotations is best. Each one possesses its advantages and drawbacks, with respect to the intended application. Hence, it is likely that several parametrizations be used simultaneously, with conversions between them. For example, the unit quaternion is ideally suited 
for interpolation [11], while the axis-angle vector is more appropriate for differential control with inverse kinematics [4]. Euler angles would not be a good choice in both applications. Instead, they are a more intuitive set of parameters to manipulate a ball-and-socket joint in a graphical user interface.

An important point to consider when comparing two parametrizations is the presence of singularities. Singularities are locations in the parameter space that result in the same orientation of the joint. Sometimes these singularities are purely mathematical and only result from the choice of parametrization, but they may also reflect a physical reality. In that case, we encounter the problem known as gimbal lock [11, 4]. Because of the problems induced by the singularities not only at the singular point but also in their neighborhood, the configuration of a joint should always be kept as far as possible from these points.

It is well-known that any three-dimensional parametrization of rotations presents at least one singularity [8]. Those of the Euler angles are discussed in [4], and will be recalled later. The unit quaternion parametrization is singularity-free, but at the cost of requiring four parameters instead of three, and a quadratic constraint (unit norm) must then be ensured [4].

\subsection{Parametrization for range of motion definition}

For the purpose of defining a range of motion, an appropriate parametrization is needed. Certainly, one can impose limits on any parametrization. For example, it is possible to impose limits on Euler angles or on quaternion parameters. For example, Lee [6] describes simple analytical constraints (such as axial, spherical or conical constraints) enforced directly in quaternion space. More complex constraints can then be defined by combining the simple ones with boolean operators. While simple and elegant, this method is not precise enough for an accurate modelling of the limits of complex joints such as the shoulder, and placing more complex meaningful limits on quaternions is difficult.

To simplify the problem, the joint limits may be decoupled. For example, independent limits may be specified on each Euler angle, or on each element of the axis-angle. However, the resulting range of motion can hardly match real motion ranges with sufficient precision [7].

For the purpose of defining a range of motion, neither the axis-angle nor the unit quaternion reflect the intuitive decomposition of the rotation into a swing and a twist component. Euler angles do, since the third angle may be used to perform the twisting motion. However, in the following sections we see that the first two Euler angles can be replaced by an axis-angle vector 
with zero component along the $z$ axis: this alleviates the problem of singularities that affects the Euler angles.

\subsection{The swing and twist decomposition of an orientation}

Intuitively, the orientation $R$ of a ball-and-socket joint can be thought as being composed of a swing component, that controls the direction of the limb directly attached to it, and a twist component that lets the limb rotate about itself [5, 4]. This may be written as: $R=R^{\text {twist }} R^{\text {swing }}$

The twist component is easily parametrized by a single angle of rotation, noted $\tau$ : hence, $R^{t w i s t}=R_{z}(\tau)$. However, this rotation must be done with respect to a well-defined orientation, here called the zero twist reference orientation. In fact, this reference orientation merely results from the swing rotation, and is not necessarily a good reference. Hence a relative twist, $\tau_{\text {offset }}$, as a function of the swing parameters, can be added. An example of such an offset function is given by Badler [1].

The purpose of the swing motion is to orientate the outgoing limb in a prescribed direction given by a unit vector $\boldsymbol{d}$. To transform the $\mathbf{z}$ vector into the $d$ vector, a rotation matrix $R^{\text {swing }}$ must be defined. We consider two solutions.

The first is to perform two successive rotations, for instance one about the $x$ axis and then a second one about the rotated $y$ axis: $R^{\text {swing }}=R_{y}(\beta) R_{x}(\alpha)$. This is equivalent to the first two rotations of the ZYX Euler angles sequence [8] (Fig. 3).

The second is to perform a single, direct rotation: $R^{\text {swing }}=R_{D}(z, d)$ (Fig. 3). Note that the axis of rotation always lies in the $\boldsymbol{x}-\boldsymbol{y}$ plane. This solution has been used by Korein [5] and Grassia [4].

As already noted by Korein [5], the difference between the two solutions lies in the final twist about the $\boldsymbol{d}$ axis, which is given by the different orientations of the rotated $\boldsymbol{x}$ and $\boldsymbol{y}$ vectors. Table 1 shows a sampling of the zero twist on the sphere for the two parametrizations: the outgoing arrow at each point on the sphere indicates the direction of the rotated $\boldsymbol{x}$ axis, which is taken as a reference to indicate the twist.

As said before, the singularities of a parametrization must also be considered, because the presence of singularities may be problematic for several applications. For the purpose of defining a range of motion, the twist component is affected by a singularity of the swing component: for example, no zero twist may be defined at a singularity, since an infinity of twists are possible. An arbitrary twist may be assigned to this point, but there is still a discontinuity with respect to its neighborhood. Table 1 compares the position of the singularities on the sphere, and the next two sections discuss and compare these singularities. 

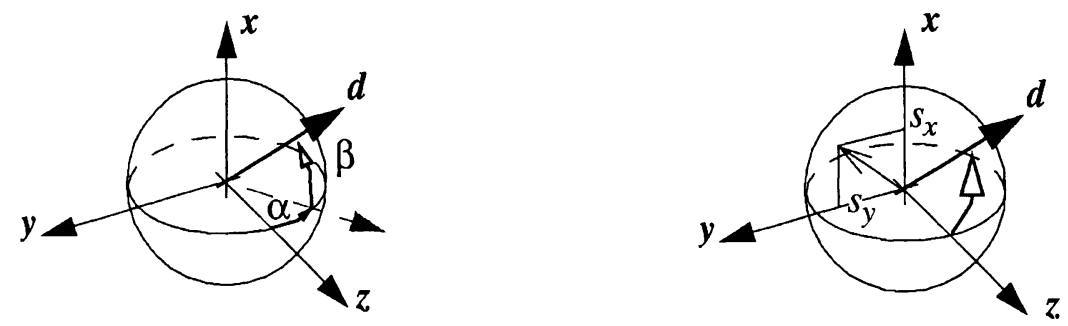

Figure 3. Euler angles parametrization (left) and axis-angle (right) of the swing motion.

\subsection{Singularities of the XY Euler angles parametrization}

This parametrization possesses two singularities: one at $\beta=\pi / 2$ and another at $\beta=-\pi / 2$. In Cartesian space, these singularities correspond to directions $d_{l}=\left[\begin{array}{lll}1 & 0 & 0\end{array}\right]^{\mathrm{T}}$ and $d_{2}=\left[\begin{array}{lll}-1 & 0 & 0\end{array}\right]^{\mathrm{T}}$ respectively, and any twist is possible there. Furthermore, moving close to these directions results in wild variations of twist. For example, moving along a closed path close to, and around the singularity, results in a complete rotation of the segment about itself (i.e. a twist of $2 \pi$ radians).

To understand the meaning of the singularities, consider a universal joint, made as a sequence of two revolute joints whose axes of rotation are orthogonal, as shown in Fig. 4. A rotation about the $\boldsymbol{x}$ axis or the $\boldsymbol{y}$ axis changes the direction of the outgoing segment, and apparently no twisting is performed. However, this is not always true. When $\beta= \pm \pi / 2$, which is the angle of rotation about the $y$ axis, the outgoing segment becomes aligned with the $\boldsymbol{x}$ axis (Fig. 5): as a consequence, a change in $\alpha$ does not change its direction anymore, but its twist. Actually, any twist is possible in this direction, but the segment cannot move up and down anymore. This phenomenon is known as gimbal lock, and is a well-known flaw of Euler angles [11]. Also note how the vertical swing component (along the $\boldsymbol{x}$ axis) gradually transforms into a twist of the outgoing limb, as the singular configuration is approached. This shows that the problem not only exists at the singularity, but also in its vicinity. 
Table 1. Comparison of "zero" twist and singularities $(\mathbf{0})$ for two parametrizations of swing.

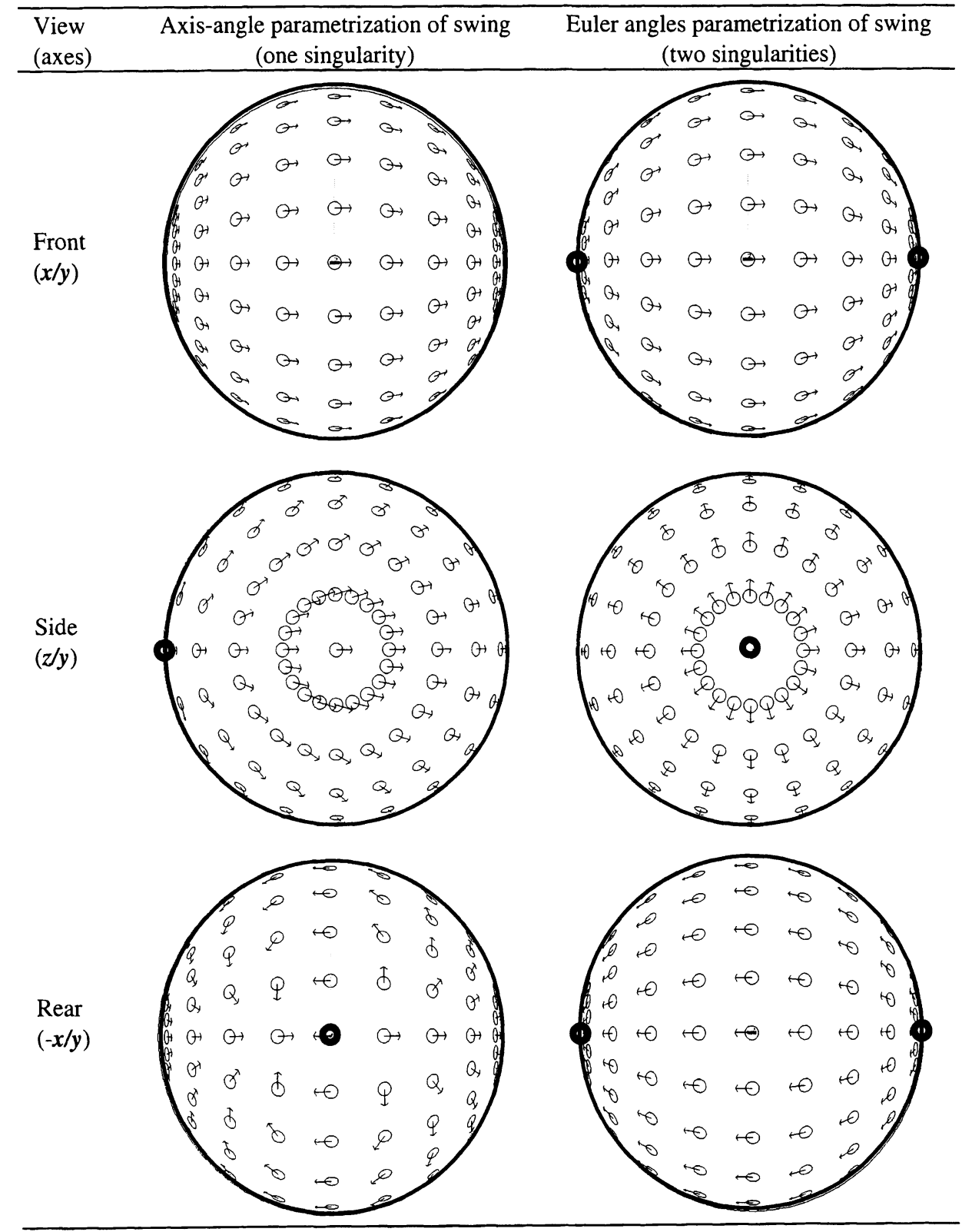




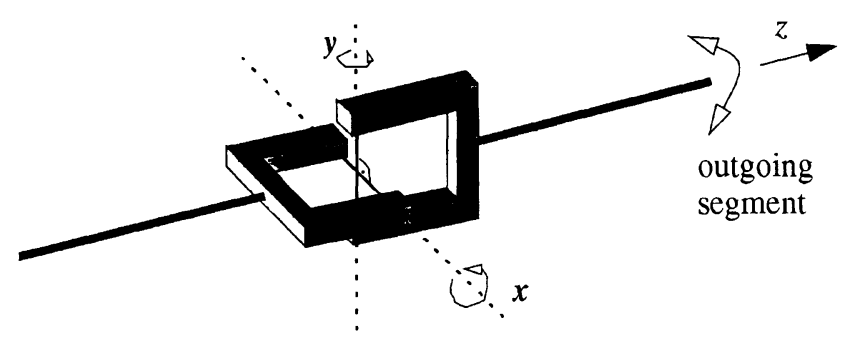

Figure 4. Illustration of the universal joint, with two orthogonal rotation axes.

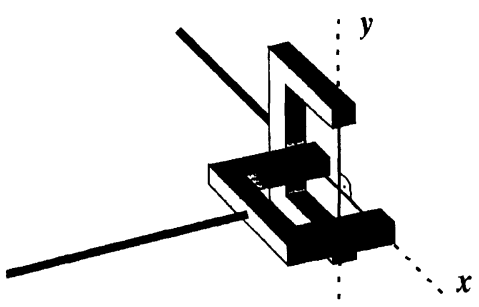

Figure 5. The universal joint in a singular configuration $(\beta=\pi / 2)$.

\subsection{Singularity of the axis-angle swing paramatrization}

The axis-angle possesses only one singularity on direction $\boldsymbol{d}=\left[\begin{array}{lll}0 & 0 & -1\end{array}\right]^{\mathrm{T}}$, where $s_{x}{ }^{2}+s_{y}{ }^{2}=\pi^{2}$. Again, any twist is possible there. However, this singularity is more "severe" since a closed path close to, and around the singularity, performs two complete rotations of the segment (i.e. a twist of $4 \pi$ radians).

To summarize, the axis-angle parametrization is preferable to the Euler angles parametrization, since it is easier to avoid one single singular point than two antipodal singular points on the sphere. To stay as far as possible from the singularity, the motion range should be centered about the $z$ axis in its "zero" configuration, or at least the singular point should not be part of the motion range.

\section{THE DEFINITION OF JOINT LIMITS}

Based on the swing and twist decomposition, independent limits can be imposed on both components. The limits of the swing component are best visualized as a curve on a sphere centered at the joint center. This curve delineates the valid region for the limb, and can be seen as the directrix of a 
general conical surface whose vertex is the center of the joint. In the following two sub-sections, we review two possible methods for defining this curve. The third section discusses the limits of the twist component.

\subsection{Swing function : the spherical ellipse}

An analytical method is to use a function $f\left(s_{x}, s_{y}\right)$ which is negative only for valid swings $\left[s_{x} s_{y}\right]^{T}$. A simple example given in [4] is an ellipse with semi-axes $r_{x}$ and $r_{y}$, that describe the maximum angle of rotation around the $\boldsymbol{x}$ axis and the $\boldsymbol{y}$ axis respectively: in this case, the function is given by $f\left(s_{x}, s_{y}\right)=\left(s_{x} / r_{x}\right)^{2}+\left(s_{y} / r_{y}\right)^{2}-1$, with $r_{x}<\pi$ and $r_{y}<\pi$. This results in a "spherical" ellipse in the Cartesian space (see Fig. 6).

The advantage of the spherical ellipse is that, with a minimum of parameters, a meaningful boundary can be defined for the swing component.
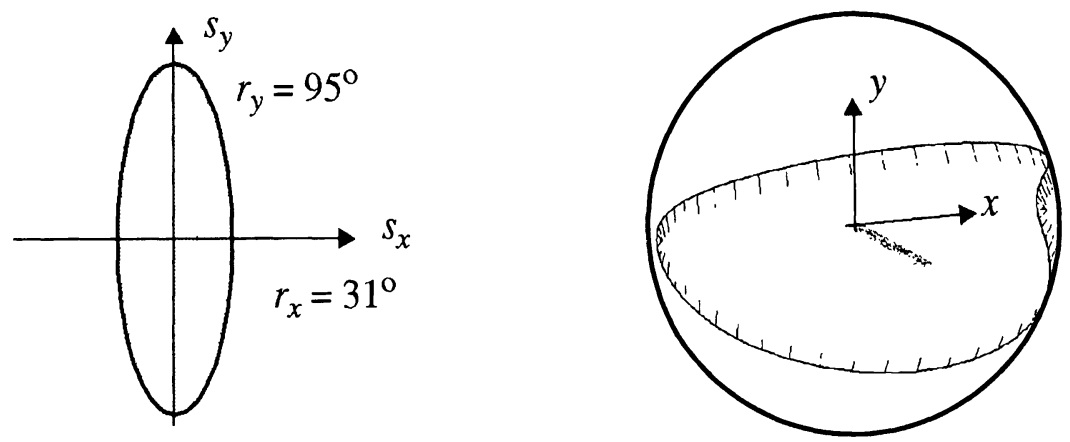

Figure 6. An example of spherical ellipse (the ticks indicate the inside region).

\subsection{Spherical polygons}

In his excellent book, Korein [5] uses a spherical polygon as directrix for the limiting cone. The edges of the spherical polygon are great arcs connecting its vertices lying on a unit sphere, and specified by three Cartesian coordinates. A great arc is the shortest path that binds two points on a sphere (it is a geodesic). The order of the vertices defines an inside region: inverting this order swaps the inside and outside regions of the polygon. Korein described an algorithm to test the inclusion of a point lying on the sphere within an arbitrary (possibly concave) spherical polygon.

Of course, spherical polygons are more general than spherical ellipses. They are also more complex to deal with. A similar method has been used by Maurel [7], but with planar polygons. As a consequence, the possible motion ranges are less general than those obtained with spherical polygons. 
However they may suffice for the human joints, and the point-in-planarpolygon test algorithm is much simpler than its spherical counterpart.

\subsection{Twist limits}

The twist motion possesses a single degree of freedom, parametrized by the angle of rotation $\tau$ about the outgoing segment. The important point is that that limits are relative to the zero twist resulting from the swing motion.

In the following globographic representations, the twist range of motion is visualized as a circular arc: it indicates the orientations that can take the reference vector (which is the $\boldsymbol{x}$ basis vector of the joint frame).

\section{AN EXAMPLE OF SHOULDER BOUNDARY WITH SWING AND TWIST COMPONENTS}

Fig. 7 shows two boundaries for the shoulder complex, based on a spherical ellipse on the left and on a spherical polygon on the right. The distal segment (the arm) is shown in its default position. The twist limits are constant over the range of swing motion (the twist motion range is about $\left.105^{\circ}\right)$. However, in reality the twist limits depend on the position of the arm, and the range can vary between $104^{\circ}$ and $160^{\circ}$ on average [10]. The data for the spherical polygon, are obtained from the results of Engin [3].
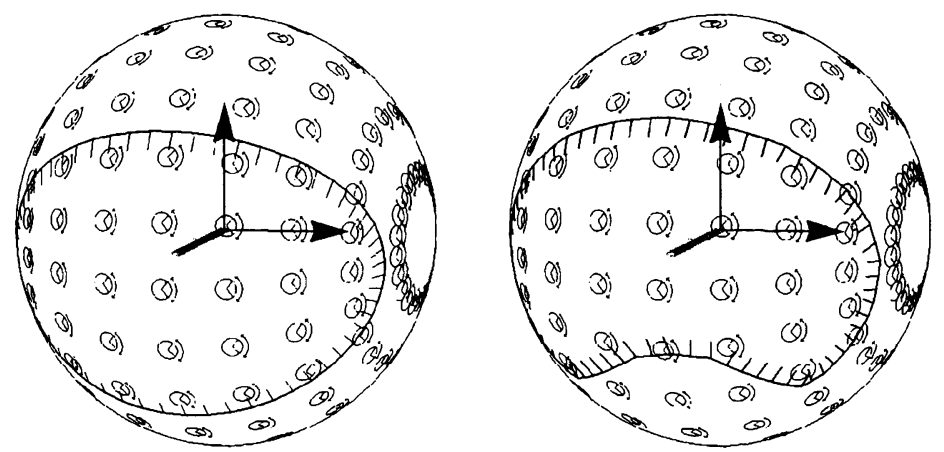

Figure 7. Shoulder motion range: spherical ellipse (left) and spherical polygon (right).

\section{CONCLUSION}

The swing-and-twist parametrization is a good basis for the definition of simple yet meaningful joint limits for ball-and-socket joints. This decomposition has been previously discussed by Korein and Grassia. In this 
paper, we have emphasized and illustrated the difference between the wellknown Euler angles parametrization and the swing-and-twist parametrization.

We have reviewed and illustrated two methods to set joint limits on the swing and twist components. The spherical ellipse proposed by Grassia is a good compromise between simplicity and accuracy, while spherical polygons are more complex but can better match real limits.

\section{ACKNOWLEDGEMENTS}

Research supported by the Swiss National Foundation for Scientific Research, grant 20-53809.98.

\section{REFERENCES}

[1] N. Badler, J. O'Rourke and B. Kaufman, "Special problems in human movement simulation", Computer Graphics (SIGGRAPH 80), 14(3), pp. 189 - 197, 1980.

[2] N. Badler, C. Phillips, B. Webber, Simulating Humans, Computer Graphics Animation and Control, New York, Oxford University Press, 1993.

[3] A.E. Engin, S.T. Tuemer, "Three-dimensional kinematic modelling of human shoulder complex - Part I: Physical model and determination of joint sinus cones", Journal of Biomechanical Engineering, 111, pp. 107 - 112, 1989.

[4] F.S. Grassia, "Practical Parameterization of Rotations using the Exponential Map", Journal of Graphics Tools, 3(3), pp. 29 - 48, 1998.

[5] J.U. Korein, "A Geometric Investigation of Reach", The MIT Press, Cambridge, 1985.

[6] J. Lee, A Hierarchical Approach to Motion Analysis and Synthesis for Articulated Figures, Ph.D. thesis, Department of Computer Science, KAIST, 2000.

[7] W. Maurel and D. Thalmann, "Human Upper Limb Modeling including Scapulo-Thoracic Constraint and Joint Sinus Cones", Computers \& Graphics, 24(2), pp. 203 - 218, 2000.

[8] R. Murray, Z. Li, S. Sastry, A mathematical introduction to robotic manipulation, CRC Press, 1994.

[9] X. Wang and J.-P. Verriest, "A geometric algorithm to predict the arm reach posture for computer-aided ergonomic evaluation", Journal of Visualization \& Computer Animation, 9, pp. 33 - 47, 1998.

[10] X.G. Wang, F. Mazet, N. Maia, K. Voinot, J.P. Verriest and M. Fayet, "Threedimensional modelling of the motion range of axial rotation of the upper arm", Journal of biomechanics, 31(10), pp. 899 - 908, 1998.

[11] A. Watt, M. Watt, Advanced Animation and Rendering Techniques, Addison-Wesley, ACM Press, 1992. 